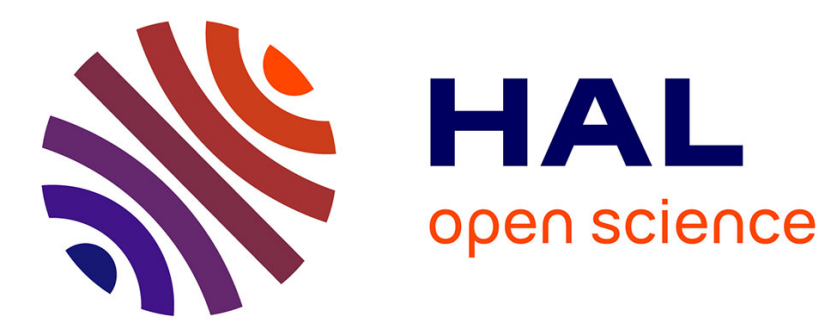

\title{
Knowledge from markers in watershed segmentation
}

Sébastien Lefèvre

\section{To cite this version:}

Sébastien Lefèvre. Knowledge from markers in watershed segmentation. 12th International Conference on Computer Analysis of Images and Patterns (CAIP 2007), 2007, Austria. pp.579-586, 10.1007/9783-540-74272-2_72. hal-00515912

\section{HAL Id: hal-00515912 \\ https://hal.science/hal-00515912}

Submitted on 8 Sep 2010

HAL is a multi-disciplinary open access archive for the deposit and dissemination of scientific research documents, whether they are published or not. The documents may come from teaching and research institutions in France or abroad, or from public or private research centers.
L'archive ouverte pluridisciplinaire HAL, est destinée au dépôt et à la diffusion de documents scientifiques de niveau recherche, publiés ou non, émanant des établissements d'enseignement et de recherche français ou étrangers, des laboratoires publics ou privés. 


\title{
Knowledge from Markers in Watershed Segmentation
}

\author{
Sébastien Lefèvre \\ LSIIT, CNRS / University Louis Pasteur - Strasbourg I \\ Parc d'Innovation, Bd Brant, BP 10413 \\ 67412 Illkirch Cedex, France \\ lefevredlsiit.u-strasbg.fr
}

\begin{abstract}
Due to its broad impact in many image analysis applications, the problem of image segmentation has been widely studied. However, there still does not exist any automatic segmentation procedure able to deal accurately with any kind of image. Thus semi-automatic segmentation methods may be seen as an appropriate alternative to solve the segmentation problem. Among these methods, the marker-based watershed has been successfully involved in various domains. In this algorithm, the user may locate the markers, which are used only as the initial starting positions of the regions to be segmented. We propose to base the segmentation process also on the contents of the markers through a supervised pixel classification, thus resulting in a knowledge-based watershed segmentation where the knowledge is built from the markers. Our contribution has been evaluated through some comparative tests with some state-of-the-art methods on the well-known Berkeley Segmentation Dataset.
\end{abstract}

Key words: Marker-based Watershed. Supervised classification. Colour Segmentation.

\section{Introduction}

Analysis, processing and understanding of digital images often involve many different algorithms. Among them, the segmentation (which consists in generating a set of meaningful regions from an input image) is certainly one of the most crucial steps as the quality of the following procedures directly depends on the accuracy and relevance of the segmentation results. So many research works are related to the problem of image segmentation, one of the main goals being to elaborate a method both automatic (without user assistance) and generic (able to deal with any kind of images). As this objective is still unreachable, the existing approaches are either automatic or generic.

Semi-automatic segmentation techniques may not be dedicated to a given type of images. Indeed, the genericity property is ensured by the user intervention or setting. There are several ways the user can drive the segmentation procedure, among which we can cite the spatial initialisation of the algorithm, the definition of the class of interest in the images, the respective influence of the differents features to be involved (e.g. colour, texture, shape, etc). The marker-based watershed [1], a widely used enhancement of the well-known watershed algorithm [2], may be driven by the user through a spatial initialisation (i.e. the location of the "markers"). Only the marker spatial position is used in the watershed algorithm, and the marker content is completely ignored. We 
propose in this paper to increase the marker-based watershed performance by using the information related to the markers content. More precisely, we use the markers as learning sets in a supervised pixel classification procedure which results in a probability map per marker. From these probability maps is then built the relief necessary to the watershed algorithm.

The rest of this paper is organized as follows. In section 2 we will recall the markerbased watershed and present existing watershed segmentation methods relying on knowledge or prior information. Then we will describe the proposed solution in section 3 and illustrate its relevance through comparative tests in section 4. Finally, section 5 will be devoted to concluding remarks.

\section{Marker-based and Knowledge-based watershed}

The watershed transform is a very popular segmentation method, as it is computationally efficient and does not require any parameter. However it has also some drawbacks, such as the sensitivity to noise and above all oversegmentation, where the result consists in a large number of irrelevant and undesired regions. To counter these limits and to increase the accuracy and relevance of the results, it is possible to consider prior knowledge. This knowledge often consists in the number and the positions of the regions through the definition of some markers, thus resulting in the marker-based watershed which will be recalled in this section. We will also present some other ways to involve knowledge in the watershed transform.

\subsection{The marker-based watershed}

The marker-based watershed [1] is certainly the mostly known and widely used enhancement of the watershed transform. Several definitions have been given in the literature, and we recall here the algorithmic definition given by Vincent and Soille [2] and called simulated immersion as it is the definition our method is relying on. In this definition, the set of the catchment basins of the greyscale image function $f$ (with values in $\left[h_{\min }, h_{\max }\right]$ is equal to the set $X_{h_{\max }}$ obtained after the following recursion:

$$
\begin{aligned}
& X_{h_{\min }}=T_{h_{\min }}(f) \\
& X_{h+1}=\operatorname{MIN}_{h+1} \cup I Z_{T_{h+1}(f)}\left(X_{h}\right), \quad h_{\min } \leq h<h_{\max }
\end{aligned}
$$

where $T_{h}$ is the threshold set at level $h, \mathrm{MIN}_{h}$ is the union of all regional minima at altitude $h$, and $I Z_{A}(B)$ is the union of geodesic influence zones of connected components of $\mathrm{B}$ defined with $B \subseteq A$ and the following equations:

$$
\begin{gathered}
I Z_{A}(B)=\bigcup_{j=1}^{l} i z_{A}\left(B_{j}\right) \\
i z_{A}\left(B_{j}\right)=\left\{p \in A \mid \forall k \in[1, l] \backslash\{j\}: d_{A}\left(p, B_{j}\right)<d_{A}\left(p, B_{k}\right)\right\} \\
d_{A}(a, B)=\min _{b \in B} d_{A}(a, b)
\end{gathered}
$$


where $d_{A}(a, b)$ represents the geodesic distance between $a$ and $b$ within $A$. The reader is refered to the original article [2] for a complete definition.

From this definition, it is possible to impose some minima to the image function $f$ at some specific locations (i.e. the markers). Let us note $M$ the set of markers, thus we can define a new image function $g$ as:

$$
g(p)= \begin{cases}h_{\min -1} & \text { if } p \in M \\ f(p) & \text { otherwise }\end{cases}
$$

where $p$ represents pixel coordinates and $h_{\min -1}$ denotes a new value dedicated for initial markers. The new recursion definition is then:

$$
\begin{aligned}
& X_{h_{\min -1}}=T_{h_{\min -1}}(g) \\
& X_{h+1}=I Z_{T_{h+1}(g)}\left(X_{h}\right), \quad h_{\min -1} \leq h<h_{\max }
\end{aligned}
$$

\subsection{Knowledge-based watershed methods}

In [3], Beare considers spatial prior knowledge and introduces a way to constrain the growing of the markers through the use of structuring element-based distance functions. The proposed method is able to deal with noisy or incomplete object boundaries.

Li and Hamarneh [4] consider a set of training images with expert-made segmentation to build shape histograms and appearance descriptors (mean and variance of the object pixel intensities). Their knowledge-based segmentation procedure relies on a classical watershed followed by a k-means clustering algorithm, but the shape and appearance information are not taken into account in the watershed segmentation step.

In a previous work [5], we have proposed to involve knowledge as labelled pixels in the watershed segmentation of remote sensed multispectral images. More precisely, a set of $c$ predefined classes (e.g. building roofs, vegetation, and roads) is considered and sample pixels are given for each class. Then a supervised fuzzy pixel classification (based on spectral signatures) is involved to generate probability maps gathered into a single $c$-band image. A morphological gradient is applied on this image and the euclidean norm is considered to obtain a graylevel image, on which the watershed transform is finally applied. So the knowledge is only related to spectral information.

To the best of the author's knowledge, the only attempt to use the markers content as a knowledge source in the watershed segmentation was done by Grau et al in [6], where a very restrictive assumption is made on normal distribution for the objects in the image. Each marker or class being represented by the mean and variance of its pixel values, they consider a Bayesian framework and model local correlations between pixels through Markov Random Fields. Thus the overall marker-based watershed process is very time-consuming.

\section{Proposed method}

In the definition of the marker-based watershed given previously, the set of markers $M$ was used only to generate the initial set $X_{h_{\min -1}}$ involved in the recursive algorithm. In this paper, we propose to use not only the position of the markers but also their content. 
Let us first modify the definition of the markers, considering from now a collection $M=\left\{M_{i}\right\}_{1 \leq i \leq c}$ of $c$ markers. Each individual marker is a set of points $M_{i}=\{p\}_{1 \leq p \leq n}$, thus resulting in either one or several connected components. These points may be characterized by various features, such as intensity, colour, spectral signature, texture, etc. We associate to each marker $M_{i}$ a class $C_{i}$ and we apply then a given supervised (soft or fuzzy) pixel classification, using $M_{i}$ as the learning set for the class $C_{i}$. The supervised classification procedure will return a set of probability values $\left\{w_{i}(p)\right\}$ where $w_{i}(p)$ represents the probability a pixel $p$ would belong to the class $i$, with the constraint $\sum_{1 \leq i \leq c} w_{i}(p)=1$. From the content of a given marker $M_{i}$, we have then generated a new image $w_{i}$ where high values represent pixels which most probably belong to $M_{i}$. As the watershed paradigm considers an increasing level $h$, we define the functions $f_{i}=\left(1-w_{i}\right) \cdot f$ where pixels with high probability $w_{i}$ will have their relative input relief $f$ lowered whereas pixels with low probability will be kept unchanged. It is also possible to consider only the probability maps (thus defining $f_{i}=1-w_{i}$ ) but we have observed in our experiments poorer results as in this case the segmentation process relies too much on the classification step. The functions $f_{i}$ will be considered as the reliefs in the watershed process.

The watershed algorithm (either standard or marker-based) relies on a grayscale image $f$. Here the supervised classification procedure results in a set of $c$ images $f_{i}$. A standard way to combine these images is to compute a given norm (such as the euclidean norm) as in [5]. In the context of marker-based watershed segmentation, it is not necessary to merge all $f_{i}$ images into a single one, and we rather consider a different image $f_{i}$ for each marker $M_{i}$. So the usual algorithm from Vincent and Soille cannot be applied directly and should be adapted to our case.

More precisely, we define the functions $g_{i}$ as

$$
g_{i}(p)= \begin{cases}h_{\min -1} & \text { if } p \in M_{i} \\ f_{i}(p) & \text { otherwise }\end{cases}
$$

and set $X=\left\{X^{i}\right\}_{1 \leq i \leq c}$, thus modifying the recursive scheme:

$$
\begin{aligned}
& X_{h_{\min -1}}^{i}=T_{h_{\min -1}}\left(g_{i}\right) \\
& X_{h+1}^{i}=I Z_{\left\{T_{h+1}\left(g_{i}\right)\right\}_{1 \leq i \leq c}}^{i}\left(X_{h}\right), \quad h_{\min -1} \leq h<h_{\max }
\end{aligned}
$$

and considering an adapted definition of the influence zones by the following equations:

$$
\begin{gathered}
I Z_{A}^{i}(B)=\bigcup_{j=1}^{l} i z_{A}^{i}\left(B_{j}^{i}\right) \\
i z_{A}^{i}\left(B_{j}^{i}\right)=i z_{A}\left(B_{j}^{i}\right) \cup \bigcap_{m=1}^{c}\left\{p \in A^{i} \cap A^{m} \mid \forall k \in[1, l]: d_{A^{i}}\left(p, B_{j}^{i}\right)<d_{A^{m}}\left(p, B_{k}^{m}\right)\right\}
\end{gathered}
$$

In other words, each catchment basin is initially defined from a given marker and will grow relying mainly on the relief built from its related marker. Many relief functions $f_{i}$ will be involved only in case of borderline pixels which could be assigned to different catchment basins. 


\section{Results}

We have evaluated our method on the freely available Berkeley Segmentation Dataset [7] which contains mainly natural colour images (animals, landscape, etc) of size $481 \times$ 321 pixels. In order to underline the potential interest of the proposed solution against the state-of-the-art, we have also considered two other well-known segmentation methods. First, as our method is relying on a supervised classification using markers as learning sets, we have performed a segmentation directly based on the classification algorithm under consideration. Each pixel is then given the class for which it has the highest probability. Second, as our method is relying on a marker-based watershed algorithm, we have obviously compared with the usual marker-based watershed segmentation, considering the same markers. Comparing our contribution with these two widely used approaches helps us to illustrate the interest of combining the marker-based segmentation watershed and the supervised pixel classification in a single procedure, thus benefiting from advantages of both techniques.

The proposed method may deal with any kind of images (grayscale, colour, multispectral) and may consider any kind of features (spectral or colour signature, texture, etc). For the sake of simplicity, we have limited ourselves here to the case of colour images where each pixel is represented by its tristimulus RGB (Red Green Blue) values. We could have used more appropriate features (texture, colour hue, etc) but let us recall that our goal is to show the potential interest of the proposed method compared to classical marker-based watershed and supervised classification, rather than to obtain optimal segmentation results for a given dataset. However, the results given in this section are rather conclusive. Compared to other related experiments made recently on the considered dataset, for instance the seed-based approach from Micusik and Hanbury [8], our method performs well without requiring neither a large number nor a precise location of markers or seeds, as illustrated by figure 2. Moreover, let us notice that our method was experimented here only with RGB values whereas results from [8] benefit from more elaborated features (brightness, colour and texture).

There are only a few parameters used in the comparison process. A $K$-nearest neighbour algorithm is involved for pixel classification purpose (for both classificationbased segmentation and our method), with the following parameters: $K=5$, a distance weighting scheme, a number of learning samples per class being equal for all classes and less or equal to 100 . For the classical watershed algorithm, we use a morphological gradient (defined as the difference between a dilation and an erosion) with a squared structural element of size $3 \times 3$ pixels and an euclidean norm in the RGB space to create the relief on which the watershed algorithm would be applied.

Figure 1 shows the segmentation results obtained for two different images with a common depth of field property. As we can notice on the left images, the two markers (for the object and the background) are rather small and far from the actual object edges. Thus the classical marker-based watershed is unable to segment correctly the objects. The 2 class supervised classification brings interesting results, as the edges between objects are clearly visible, but it is hard to determine the appopriate object edges from the markers and the classification map. The proposed approach is far more accurate due to the fact it takes into account both the spatial positions of the markers (as initial 
positions of the catchment basins) and the colour content of the markers (as learning sets of the classification procedure).

The number of markers is variable and it could be more relevant to use in some cases more than two markers. For instance, we compare in figure 3 the initialisation by two markers and by three markers, considering respectively only one or two markers for the background. In the last case, using two markers for the background helps to increase the accuracy of the supervised classification procedure by considering two distinct classes instead of only one. Moreover, we can see here the ability of the method to correctly separate the field and the wood.

A complete set of segmentation results is given in figure 4 . We can notice that the proposed approach returns often accurate results, except for the two last images where the learning procedure returns poor results, thus resulting in an inaccurate segmentation using our method.

The computational cost of the proposed approach is of course higher than the classical marker-based watershed as it also involves a supervised pixel classification. However, it is still reasonable and as been measured around 15 seconds with a Java-based implementation on a Pentium M 1.1 GHz / 1 GB RAM laptop.
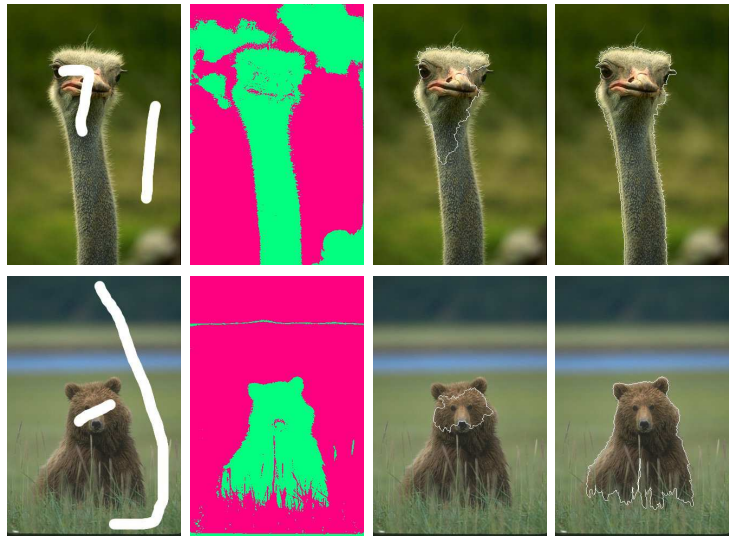

Fig. 1. Comparative results (from left to right): input image with markers, classical supervised pixel classification, classical marker-based watershed segmentation, and proposed approach.

\section{Conclusion}

In this paper we dealt with the problem of image segmentation, for which the markerbased watershed algorithm has been one of the most widely accepted solutions. This algorithm requires a spatial initialisation made either automatically or manually to define the initial position of the catchment basins (i.e. the markers). However the use made of this very relevant information is rather limited. 

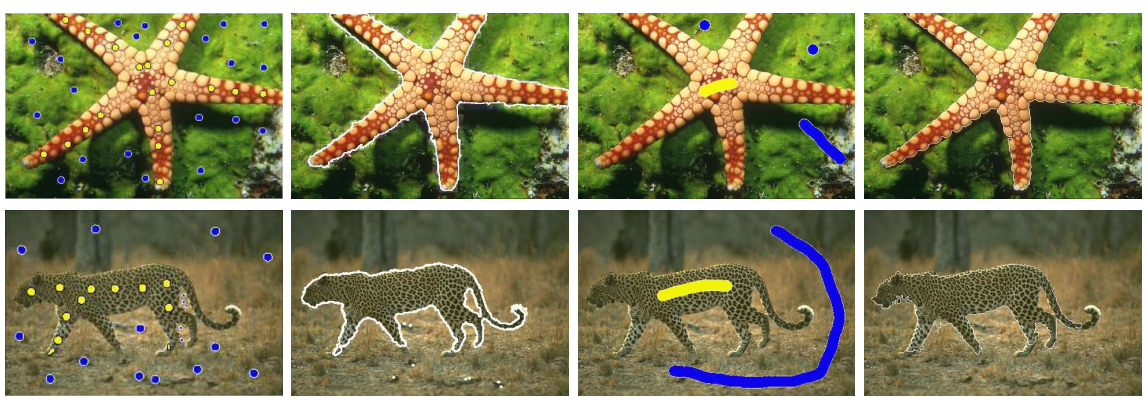

Fig. 2. Comparison between [8] and our approach (from left to right): initial seeds and segmentation result for [8], markers and segmentation result for our approach.

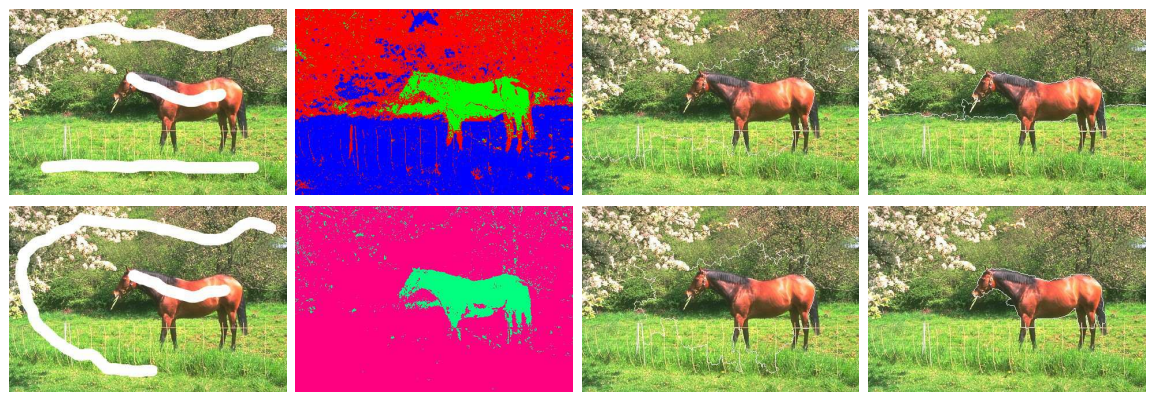

Fig. 3. Influence of the number of markers on the results (from left to right): input image with markers, classical supervised pixel classification, classical marker-based watershed segmentation, and proposed approach.
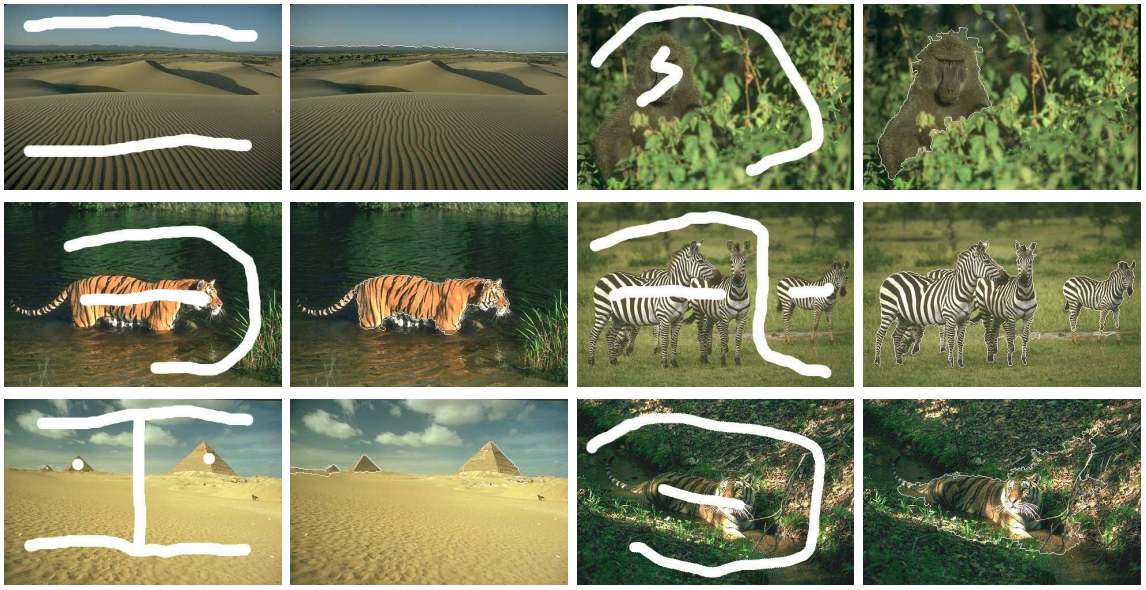

Fig. 4. Segmentation results on various images (markers and results). 
So we proposed here to use the markers as learning sets in a supervised pixel classification procedure. Thus we obtain, for each marker or class, the probability for each pixel to belong to the given class through a probability map. It is then possible to build from this map the relief necessary to the marker-based watershed algorithm, and to make use of both the spatial positions and the contents of the markers. We made some conclusive tests on the Berkeley segmentation dataset.

In order to better evaluate the segmentation results returned by our method, we consider to measure their relevance and accuracy using the user-made references results from the Berkeley segmentation dataset [7]. Moreover, we will involve other features, related either to texture $[9,10]$ or colour [11], and other classifiers (e.g. support vector machines). The robustness of the method to the initial location of the markers should also be evaluated in order to determine the minimal marker size.

A major improvement of the method could finally be achieved by following the idea from Micusik and Hanbury who have built a completely automatic segmentation solution [12] by iterating their semi-automatic segmentation method [8], thus removing the need for manual setting of the markers.

\section{References}

1. Rivest, J., Beucher, S., Delhomme, J.: Marker-controlled segmentation: an application to electrical borehole imaging. Journal of Electronic Imaging 1(2) (April 1992) 136-142

2. Vincent, L., Soille, P.: Watersheds in digital spaces: An efficient algorithm based on immersion simulations. IEEE Transactions on Pattern Analysis and Machine Intelligence 13(6) (June 1991) 583-598

3. Beare, R.: A locally constrained watershed transform. IEEE Transactions on Pattern Analysis and Machine Intelligence 28(7) (July 2006) 1063-1074

4. Li, X., Hamameh, G.: Modeling prior shape and appearance knowledge in watershed segmentation. In: Canadian Conference on Computer Vision. (2005)

5. Derivaux, S., Lefèvre, S., Wemmert, C., Korczak, J.: Watershed segmentation of remotely sensed images based on a supervised fuzzy pixel classification. In: IEEE International Geosciences And Remote Sensing Symposium, Denver, USA (July 2006)

6. Grau, V., Mewes, A., Alcaniz, M., Kikinis, R., Warfield, S.: Improved watershed transform for medical image segmentation using prior information. IEEE Transactions on Medical Imaging 23(4) (April 2004) 447-458

7. Martin, D., Fowlkes, C., Tal, D., Malik, J.: A database of human segmented natural images and its application to evaluating segmentation algorithms and measuring ecological statistics. In: IEEE International Conference on Computer Vision. Volume 2. (July 2001) 416-423

8. Micusik, B., Hanbury, A.: Steerable semi-automatic segmentation of textured images. In: Scandinavian Conference on Image Analysis. (2005)

9. Aptoula, E., Lefèvre, S.: Spatial morphological covariance applied to texture classification. In: International Workshop on Multimedia Content Representation, Classification and Security (IWMRCS), Istanbul, Turkey (September 2006) 522-529

10. Lefèvre, S.: Extending morphological signatures for visual pattern recognition. In: IAPR International Workshop on Pattern Recognition in Information Systems. (June 2007)

11. Aptoula, E., Lefèvre, S.: A comparative study on multivariate mathematical morphology. Pattern Recognition (January 2007) To appear.

12. Micusik, B., Hanbury, A.: Automatic image segmentation by positioning a seed. In: European Conference on Computer Vision. (2006) 